\title{
ERRATUM
}

\section{Erratum to: Prevalence of renal artery stenosis in patients undergoing cardiac catheterization}

\author{
Carmelita Marcantoni - Stefania Rastelli - Luca Zanoli • Giovanni Tripepi • \\ Marilena Di Salvo • Sergio Monaco • Carmelo Sgroi - Davide Capodanno • \\ Corrado Tamburino $\cdot$ Pietro Castellino
}

Published online: 26 June 2011

(c) SIMI 2011

\section{Erratum to: Intern Emerg Med}

DOI 10.1007/s11739-011-0624-5

Unfortunately, first and last name of each author has been swapped in the original version of the article.

The correct author group is listed below:

Carmelita Marcantoni, Stefania Rastelli, Luca Zanoli, Giovanni Tripepi, Marilena Di Salvo, Sergio Monaco, Carmelo Sgroi, Davide Capodanno, Corrado Tamburino, Pietro Castellino.

The online version of the original article can be found under doi: 10.1007/s11739-011-0624-5.

C. Marcantoni $(\square)$

Division of Nephrology, Cannizzaro Hospital, Catania, Italy

e-mail: carmelita.marcantoni@gmail.com

S. Rastelli · L. Zanoli · P. Castellino

Internal Medicine, University of Catania, Catania, Italy

G. Tripepi

CNR-IBIM, Reggio, Calabria, Italy

M. Di Salvo - S. Monaco - C. Sgroi - D. Capodanno ·

C. Tamburino

Division of Cardiology, University of Catania, Catania, Italy 